\title{
Linfangioma mesentérico quístico de intestino delgado simulando tumoración ovárica
}

\author{
Mesenteric cystic lymphangioma of small bowel mimicking \\ ovarian tumor
}

Ismael Suárez-Torres' * , Eduardo Reyna-Villasmil², Duly Torres-Cepeda²

Fecha correspondencia:

Recibido: octubre 31 de 2016.

Revisado: mayo 8 de 2017.

Aceptado: julio 27 de 2017.

Forma de citar:

Suarez-Torres I, Reyna-Villasmil

E, Torres-Cepeda D. Linfangioma mesentérico quístico de intestino delgado simulando tumoración ovárica. CES Med 2017; 31(2):

215-221.

\author{
Open access \\ (C) Derecho de autor \\ Licencia creative commons \\ Ética de publicaciones \\ Revisión por pares \\ Gestión por Open Journal System \\ DOl: http://dx.doi.org/10.21615/ \\ cesmedicina.31.2.11 \\ ISSN 0120-8705 \\ e-ISSN 2215-9177
}

Sobre los autores:

1 Médico cirujano. Residente Ginecología y Obstetricia. Servicio de Ginecología. Hospital Central "Dr. Urquinaona", Maracaibo, Venezuela.

\section{Resumen}

El linfangioma quístico, caracterizado por proliferación de los espacios linfáticos, es una lesión infrecuente en pacientes adultos. Menos del 1\% de todos los linfangiomas corresponden a linfangiomas del intestino delgado. La presentación clínica es diversa, variando desde un quiste abdominal asintomático, diagnosticado de forma incidental, a un cuadro de abdomen agudo. Se presenta un caso de linfangioma mesentérico quístico del intestino delgado en una mujer de 30 años quien consultó por dolor abdominal y vómitos. La ecografía y la tomografía computada abdominal mostraron un quiste con múltiples septos en el cuadrante inferior derecho simulando un tumor de ovario. En la laparotomía se encontró un tumor quístico, lobulado y marrón, procedente del mesenterio del intestino delgado. A la paciente se le realizó resección de la tumoración y del intestino delgado adyacente con reconstrucción del tránsito intestinal. La evaluación histológica confirmó el diagnóstico de linfangioma mesentérico quístico.

Palabras claves: Linfangioma; Linfangioma mesentérico quístico; Tumor abdominal; Intestino delgado.

\begin{abstract}
Cystic lymphangioma, characterized by proliferation of lymphatic spaces, is an infrequent lesion in adult patients. Less than $1 \%$ of all lymphangiomas correspond to small-bowel lymphangiomas. Clinical presentation can be diverse ranging from incidental abdominal cysts to an acute abdomen. We report a case of small bowel mesenteric cystic lymphangioma in a 30 yearsold woman who presented with abdominal pain and vomiting. The abdominal ultrasound and computed tomography scan showed a cystic multiseptated mass at the right lower quadrant mimicking an ovarian tumor. Laparotomy found a lobulated, brown, cystic tumor from the small bowel mesentery. The patient underwent resection of the tumor and the adjacent small intestine with reconstruction of the intestinal transit. The histological study confirmed diagnosis of mesenteric cystic lymphangioma.
\end{abstract}

Keywords: Lymphangioma; Mesenteric cystic lymphangioma; Abdominopelvic mass; Small bowel. 
Julio - Diciembre 2017 - Pág 215

2 Doctor en Ciencias Médicas. Especialista en Ginecología y Obstetricia. Servicio de Ginecología. Hospital Central "Dr. Urquinaona", Maracaibo, Venezuela.

Los linfangiomas quísticos son tumores benignos, de crecimiento lento, derivados del tejido linfático y ocurren principalmente en niños en cuello y axila.

\section{Introducción}

Los linfangiomas quísticos son tumores benignos, de crecimiento lento, derivados del tejido linfático y ocurren principalmente en niños, en la región del cuello y axila. La localización intraabdominal es rara y pueden presentarse en retroperitoneo, tracto gastrointestinal o mesenterio de los órganos abdominales $(1,2)$. Los linfangiomas mesentéricos quísticos del intestino delgado son raros y representan $1 \%$ de los reportes $(2,3)$.

Se presenta un caso de un linfangioma mesentérico quístico del intestino delgado simulando una tumoración ovárica.

\section{Caso clínico}

Se trataba de una paciente de 30 años quien consultó por presentar varios episodios de dolor abdominal vago e inespecífico de moderada intensidad en hipogastrio y ambas fosas iliacas, acompañado de vómitos de una semana de evolución. La paciente negaba fiebre, alteraciones miccionales o evacuatorias. También negaba antecedentes personales de afecciones médicas o quirúrgicas.

Los exámenes hematológicos y bioquímicos estaban dentro de límites normales. El examen clínico no mostró ninguna alteración. El examen pélvico bimanual evidenció la presencia de una tumoración palpable, móvil en el anexo derecho. El ultrasonido abdomino-pélvico mostró tumoración con paredes finas y múltiples septos finos, en el anexo derecho, que medía 25 centímetros de diámetro y que fue diagnosticado como tumor de ovario (figura 1). La resonancia magnética mostró tumor quístico multiloculado de $25 \times 20 \times 16 \mathrm{~cm}$ en el fondo de saco de Douglas que se extendía a ambos lados del útero, sin evidencia de líquido libre intraperitoneal, estableciendo la posibilidad diagnóstica de tumoración quística ovárica.

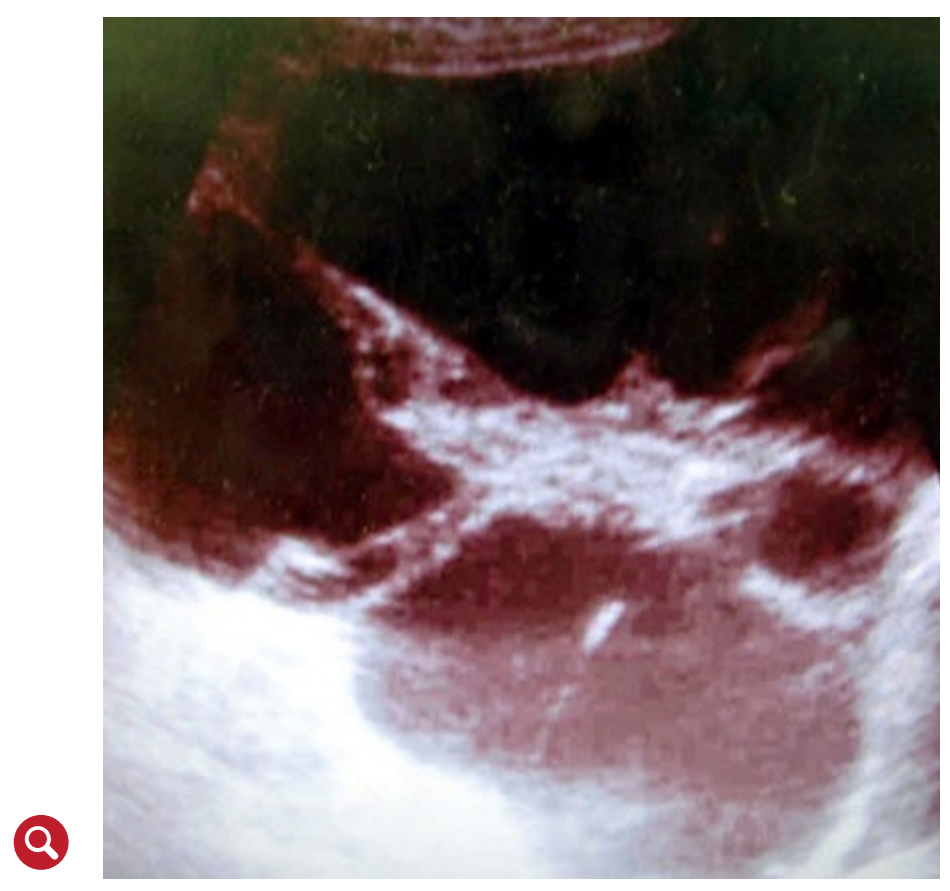

Figura 1. Imagen ecográfica del abdomen en la cual se observa la tumoración quística 
Su patogénesis es desconocida. La teoría más aceptada es la de congestión linfática por interrupción congénita en la comunicación del sistema de drenaje linfático durante el periodo embrionario.
Durante la laparotomía se observó un tumor quístico de aproximadamente 24 x 20 x 15 centímetros con paredes gruesas, multiloculado, lleno de líquido marrón espeso que se originaba del mesenterio del intestino delgado, el cual fue resecado junto a una porción del intestino delgado al cual estaba incluido (figura 2). Se revisó el resto del abdomen y los órganos intraabdominales sin encontrar inclusiones neoplásicas u otras alteraciones, realizándose reconstrucción del tránsito intestinal mediante anastomosis termino-terminal del intestino delgado.

El estudio histológico demostró vasos linfáticos dilatados rodeados por células endoteliales con linfocitos dispersos confirmando el diagnóstico de linfangioma mesentérico quístico. La mucosa del intestino delgado presentaba características normales sin invaginaciones dentro de la tumoración. Los exámenes de inmunoreactividad fueron positivos para CD31 y D2-40 y negativo para calretinina.

El postoperatorio de la paciente no presentó complicaciones y fue dada de alta al quinto día. La paciente se mantiene asintomática seis meses después de la cirugía.

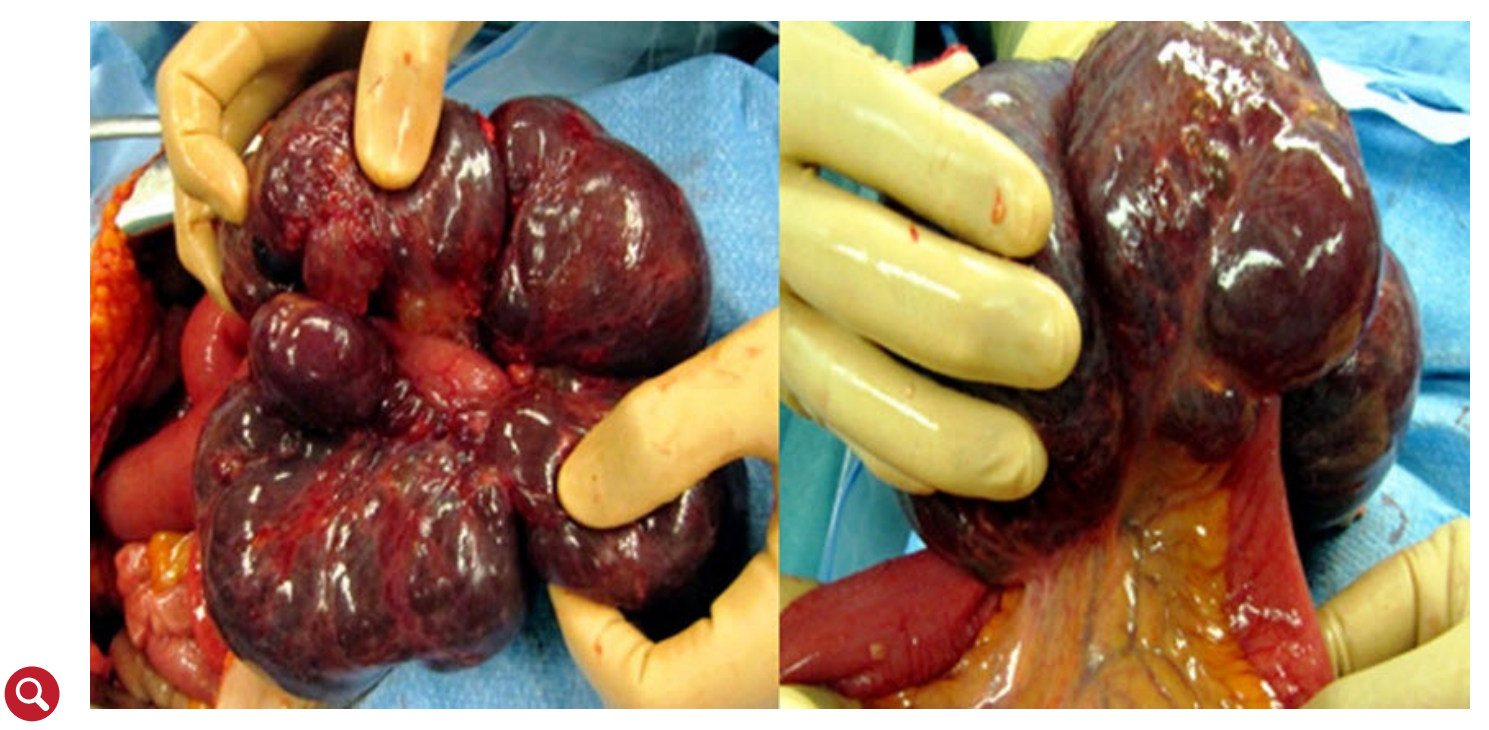

Figura 2. Imagen intraoperatoria en la que se observa el linfangioma mesentérico quístico junto con la porción de intestino delgado adyacente

\section{Discusión}

Los linfangiomas quísticos son infrecuentes y ocurren principalmente en la infancia y sus sitios de presentación más común son la cabeza, el cuello y la región axilar (4). En forma ocasional también se puede diagnosticar en el adulto en diferentes sitios anatómicos. El mesenterio del intestino delgado $(70 \%)$ es la localización intraabdominal más común, con 50-60 \% de los quistes localizados en el mesenterio del íleo (ㅁ).

Tiene una prevalencia aproximada de 1/20 000 a 1/ 250 000; es más frecuente en hombres que en mujeres con una relación de 3:1 y el $90 \%$ de los casos son diagnosticados antes de los dos años (ㅁ-요). Existen escasos reportes con relación a la presencia de linfangioma mesentérico quístico en adultos (9).

Su patogénesis es desconocida. La teoría más aceptada es la de congestión linfática por interrupción congénita en la comunicación del sistema de drenaje linfático durante el periodo embrionario. Algunos autores han propuesto que las manifestacio- 
La presentación clínica es variable, no existiendo signos síntomas patognomónicos, pudiendo ir desde una tumoración abdominal descubierta de forma incidental hasta la presentación de un cuadro de abdomen agudo, obstrucción intestinal, signos de peritonitis e inflamación abdominal crónica. nes en adultos son secundarias al retraso en la proliferación congénita o adquirida de los nidos linfáticos después de una infección respiratoria, inflamación, cirugía abdominal, radioaciones o trauma local (10).

Desde el punto de vista histológico, los linfangiomas se clasifican en tres tipos: capilar simple, cavernoso y quístico. Los capilares simples generalmente se encuentran superficiales en la piel y están compuestos por linfáticos de paredes finas. Los cavernosos consisten en linfáticos más grandes que tienen conexiones con linfáticos normales. Los linfangiomas quísticos consisten en espacios linfáticos de diferentes tamaños rodeados de colágeno y músculo liso que contienen líquido seroso, quiloso, sanguíneo o purulento sin conexiones con los vasos linfáticos normales adyacentes $(\underline{1}, \underline{2})$.

Aunque los linfangiomas mesentéricos quísticos abdominales habían sido considerados "quistes mesentéricos" existen divergencias histológicas. A diferencia de los quistes mesentéricos que se originan del tejido mesotelial, los linfangiomas están compuestos de tejido linfoide, espacios linfáticos y células espumosas (11).

La presentación clínica es variable, no existiendo signos síntomas patognomónicos, pudiendo ir desde una tumoración abdominal descubierta de forma incidental hasta la presentación de un cuadro de abdomen agudo, obstrucción intestinal, signos de peritonitis e inflamación abdominal crónica, dependiendo del tamaño de la lesión. La presentación "clásica" es una obstrucción parcial junto con una tumoración palpable y móvil. Son comunes los síntomas abdominales inespecíficos como dolor abdominal, vómitos y pérdida de peso. También se ha descrito edema de miembros inferiores por compresión de las lesiones de gran tamaño dependiendo del tamaño del tumor $(\underline{12}, \underline{13})$. En raros casos, la tumoración puede producir complicaciones agudas, incluyendo dolor abdominal severo debido al crecimiento rápido, ruptura traumática, hemorragia intraabdominal y obstrucción intestinal completa (12). El caso descrito es inusual puesto que fue diagnosticado en una mujer adulta con aparición repentina de los síntomas.

Las pruebas de laboratorio son de poca ayuda para el diagnóstico. La radiografía simple de abdomen no muestra hallazgos diagnósticos pero puede mostrar una lesión sin gas, homogénea con densidad similar al agua que desplaza los órganos intestinales alrededor de esta con algunas calcificaciones (6). La ecografía es superior a la tomografía con relación a la evaluación de la tumoración (estructura quística anecóica bien definida con paredes finas e irregulares y septos), estructuras intraquísticas, grosor de la cápsula y hemorragia interna $(\underline{7}, \underline{8})$.

La tomografía puede suministrar información pre-operatoria importante con relación al tamaño, localización anatómica y afección de los órganos adyacentes. La ecografía y la tomografía secuencial demuestran aumento progresivo de las lesiones quísticas, aumento de la ecogenicidad del líquido y engrosamiento de las paredes de la tumoración asociado con la multiplicación de los septos (ㅇ).

La resonancia magnética puede ser útil para el diagnóstico pero no puede diferenciar entre los quistes dermoides, teratomas quísticos, linfangiomas quísticos o linfoceles. Sin embargo, es más útil para terminar la extensión de la tumoración, ya que puede mostrar imágenes de baja intensidad en las diferentes fases (7). En este caso el diagnóstico diferencial con una tumoración ovárica fue difícil. 
El diagnóstico final está basado en hallazgos histológicos al demostrar extensas áreas miofibroblásticas que manifiestan los componentes linfáticos del tumor.

En los linfangiomas mesentéricos quísticos, el contenido es generalmente descrito como seroso con una densidad similar al agua. No se recomienda la realización de la biopsia percutánea debido a que su valor pronóstico es bajo por el escaso índice de celularidad de estos tumores.

El diagnóstico final está basado en hallazgos histológicos al demostrar extensas áreas miofibroblásticas que manifiestan los componentes linfáticos del tumor. El tumor está compuesto por espacios linfáticos dilatados en forma quística, los cuales están recubiertos de capaz musculares y está asociado con agregados linfoides. Los folículos linfoides y los infiltrados linfoideos en el estroma apoyan el diagnóstico. Los espacios linfáticos contienen tanto líquido claro como una gran cantidad de macrófagos espumosos y células linfoideas. Se han utilizado marcadores inmunohistoquímicos específicos para las células endoteliales linfáticas como el receptor 1 endotelial de vasos linfáticos, receptor 3 del factor de crecimiento endotelial vascular y Prox-1 (1).

El diagnóstico diferencial se realiza con los pseudoquistes pancreáticos, quistes dermoides, linfocele, quistes por equinococos, cistadenoma ovárico, quistes de duplicación entérica, mesoteliomas quísticos y ascitis (12).

La conducta quirúrgica, de acuerdo al tipo de linfangioma mesentérico quístico, se muestra en el cuadro 1. Dado que los tumores son estructuras quísticas con áreas de necrosis grasa y hemorragia, y en ocasiones llenos de líquido espeso, gelatinoso - lechoso, la resección quirúrgica completa es el tratamiento de elección para eliminar los síntomas, la posibilidad de extensión a otros órganos y prevenir complicaciones o recurrencias (14).

Existen reportes de malformaciones difusas en las que se realiza extensa resección intestinal, lo cual puede causar síndrome de intestino corto (ㅁ). La aspiración del líquido intratumoral disminuye el tamaño del quiste solo por un corto periodo de tiempo y aumenta el riesgo de infección. La resección incompleta tiene el riesgo de recurrencia del tumor de 0 - 13,6 \% (14-16). Aunque se ha descrito el abordaje laparoscópico utilizando aspiración parcial, en el presente caso se prefirió realizar laparotomía debido al gran tamaño de la tumoración.

La aspiración e inyección de agentes esclerosantes como OK-432, belomicina, doxiciclina, goma de fibrina y esteroides, pueden ser utilizados en el tratamiento de emergencia, en especial en aquellos lesiones no resecables y en las que existe un amplio compromiso de las estructuras intestinales adyacentes. Sin embargo, como tratamiento definitivo tienen una alta tasa de recurrencia y no ha demostrado ser mejor que la cirugía (ㅁ).

Cuadro 1. Clasificación, ubicación y conducta en los linfangiomas mesentéricos quísticos (6)

\begin{tabular}{ccc}
\hline Tipo & Ubicación & Conducta \\
\hline I o pediculado & Inespecífica & $\begin{array}{c}\text { Resección } \\
\text { II o sésil }\end{array}$ \\
III o retroperitoneal & Mesenterio intestinal & $\begin{array}{c}\text { Resección del mesenterio y } \\
\text { del intestino afectado }\end{array}$ \\
IV o multi-céntrico & los vasos mesentéricos, la aorta y la vena & Resección parcial o \\
cava & marzupialización
\end{tabular}

Tomado de ref. 6. 


\section{Conclusión}

Los linfagiomas mesentéricos quísticos son tumores benignos infrecuentes en adultos, con manifestaciones clínicas inespecíficas, por lo que se debe considerar como diagnóstico diferencial de abdomen agudo. El diagnóstico se realiza por imágenes y se confirma por los hallazgos histopatológicos. El tratamiento de elección es la cirugía con resección completa de la lesión para evitar las recurrencias. Aunque infrecuentes, se debe considerar en el diagnóstico diferencial de los tumores ováricos.

\section{Derecho a la privacidad y consentimiento informado}

Los autores han obtenido el consentimiento informado del paciente referidos en el artículo. Este documento obra en poder del autor de correspondencia.

\section{Financiamiento}

Los autores certifican que no han recibido apoyos financieros, equipos, en personal de trabajo o en especie de personas, instituciones públicas o privadas para la realización del estudio

\section{Conflicto de intereses}

Los autores declaran no tener ningún conflicto de intereses.

\section{Bibliografía}

1. Allen JG, Riall TS, Cameron JL, Askin FB, Hruban RH, Campbell KA. Abdominal lymphangiomas in adults. J Gastrointest Surg. 2006;10(5):746-51.

2. Tian $C$, Zheng $Y$, Ren $X$, Li B. A giant abdominal cystic tumour: Mesentery cystic lymphangioma. Dig Liver Dis. 2015 Sep;47(9):816-7. http://www.dldjournalonline.com/article/S1590-8658 \%2815\%2900337-0/abstract

3. Suthiwartnarueput W, Kiatipunsodsai S, Kwankua A, Chaumrattanakul U. Lymphangioma of the small bowel mesentery: a case report and review of the literature. World J Gastroenterol. 2012;18(43):6328-32. http://www.wjgnet.com/10079327/full/v18/i43/6328.htm

4. Gallart Aragón T, Palomeque Jiménez A, Pérez Cabrera B, López Cantarero M. Linfangioma quístico retroperitoneal. An Pediatr (Barc). 2014;80(6):407-8. http:// www.analesdepediatria.org/es/linkresolver/linfangioma-quistico-retroperito$\underline{\text { neal/S1695403313003792/ }}$

5. Protopapas A, Papadopoulos D, Rodolakis A, Markaki S, Antsaklis A. Mesenteric lymphangioma presenting as adnexal torsion: case report and literature review. J Clin Ultrasound. 2005;33(2):90-3. http://onlinelibrary.wiley.com/doi/10.1002/ jcu.20094/abstract;jsessionid=4F50D9EE291D833698B956F585DB0738.f03t03

6. Yildirim E, Dural K, Kaplan T, Sakinci U. Cystic lymphangioma: report of two atypical cases. Interact Cardiovasc Thorac Surg. 2004;3(1):63-5. http://icvts.oxfordjournals.org/cgi/pmidlookup?view=long\&pmid $=17670177$

7. Losanoff JE, Kjossev KT. Mesenteric cystic lymphangioma: unusual cause of intra-abdominal catastrophe in an adult. Int J Clin Pract. 2005;59(8):986-7. http:// onlinelibrary.wiley.com/doi/10.1111/j.1368-5031.2005.00554.x/abstract 
8. Wang J, Fisher C, Thway K. Combined mesothelial cyst and lymphangioma of the small bowel: a distinct hybrid intra-abdominal cyst. Int J Surg Pathol. 2014;22(6):547-51. http://ijs.sagepub.com/content/22/6/547.long

9. Chateil JF, Brun M, Vergnes P, Andrieu de Lewis P, Pérel Y, Diard F. Abdominal cystic lymphangiomas in children: presurgical evaluation with imaging. Eur J Pediatr Surg. 2002;12(1):13-8. https://www.thieme-connect.com/DOI/ DOl?10.1055/s-2002-25090

10. Guinier D, Denue PO, Mantion GA. Intra-abdominal cystic lymphangioma. Am J Surg. 2006 May;191(5):706-7. http://www.americanjournalofsurgery.com/article/S0002-9610\%2805\%2900828-7/abstract

11. Hornick JL, Fletcher CD. Intraabdominal cystic lymphangiomas obscured by marked superimposed reactive changes: clinicopathological analysis of a series. Hum Pathol. 2005;36(4):426-32. http://www.humanpathol.com/article/S00468177\%2805\%2900076-6/abstract

12. Kambakamba P, Lesurtel M, Breitenstein S, Emmert M, Wilhelm M, Clavien P. Giant mesenteric cystic lymphangioma of mesocolic origin in an asymptomatic adult patient. J Surg Case Rep. 2012;2012(6):4. http://jscr.oxfordjournals.org/ content/2012/6/4.long

13. Elukoti HN, Alcasoas S, Vernekar J, Hegde P, Pereira S. Mesenteric Lymphangioma Presenting as Ileal Volvulus. J Clin Diagn Res. 2015 Dec;9(12):TJ05-6. http:// www.ncbi.nlm.nih.gov/pmc/articles/PMC4717702/

14. Martín-Pérez E, Tejedor D, Brime R, Larrañaga E. Cystic lymphangioma of the lesser omentum in an adult. Am J Surg. 2010 Feb;199(2):e20-2. http://www. americanjournalofsurgery.com/article/S0002-9610 \%2809 \%2900426-7/abstract

15. Hureibi K, Sunidar OA. Mesenteric cystic lymphangioma mimicking malignancy. BMJ Case Rep. 2014;2014. http://casereports.bmj.com/content/2014/bcr-2014203560.long

16. Tsuboi M, Noda H, Watanabe F, Abe I, Nokubi M, Rikiyama T. Complete resection of a complicated huge mesenteric lymphangioma guided by mesenteric computed tomography angiography with three-dimensional reconstruction: report of a case. Int Surg. 2015 Mar;100(3):574-8. http://www.internationalsurgery.org/ doi/10.9738/INTSURG-D-14-00112.1?url ver=Z39.88-2003\&rfr id=ori \%3Arid \%3Acrossref.org\&rfr dat $=$ cr pub \%3Dpubmed\& 